\title{
Relaciones conyugales en Adolescentes de la IED Josefa María Romero de la Cruz ${ }^{1}$
}

\section{Pedagogical proposal for the identification of determinants in conjugal relationships in IED Adolescents Josefa Maria Romero de la Cruz}

\author{
DOI: http://dx.doi.org/10.17981/cultedusoc.9.3.2018.80
}

Artículo de investigación. Fecha de recepción: 15/06/2018. Fecha de aceptación: 27/11/2018

\author{
Walter Ospino-Pantoja ${ }^{2}$; \\ Mónica Acuña Rodríguez; Escilda García-Florez; \\ María Díaz-Carrillo; Marisela Polo-Rambal; Katherine Muñoz-Bolaño; \\ Vialis Flórez-Florez; Martha Alfaro Martínez y Jhon Jaramillo -Suarez ${ }^{3}$ \\ IED Josefa María Romero de la Cruz, Concordia-Magdalena (Colombia) \\ walter1329@gmail.com
}

Para citar este artículo:

Ospino-Pantoja, W., Acuña, M., García-Florez, E., Díaz-Carrillo, M., Polo-Rambal, M., Muñoz-Bolaño, K., Flórez-Florez, V., Alfaro, M. y Jaramillo-Suarez, J.(2018). Relaciones conyugales en Adolescentes de la IED Josefa María Romero de la Cruz. Cultura. Educación y Sociedad 9(3), 677-690. DOI: http://dx.doi.org/10.17981/cultedusoc.9.3.2018.80

\section{Resumen}

La vida en pareja a edades tempranas es un tema que ha cobrado gran interés en la actualidad, especialmente en las zonas rurales; razón por la cual se llevó a cabo el presente estudio, el cual se enfoca en identificar los factores influyentes de las relaciones conyugales en Adolescentes., para así diseñar estrategias que permitan disminuir esta situación en las escuelas. Se asume un abordaje cualitativo, se tomó como muestra 31 estudiantes de octavo, noveno y décimo grado de la Institución Educativa Josefa Romero de la Cruz. Dentro de los hallazgos encontrados existe falta de orientación en la etapa de la adolescencia para la toma de decisiones frente a iniciar una vida conyugal, por lo que los docentes deberán fortalecer en la práctica pedagógica sobre temas inherentes a la educación y formación en temas de educación sexual.

Palabras clave: Relaciones conyugales, adolescencia, educación sexual.

\section{Abstract}

Life as a couple at an early age is a topic that has gained great interest today, especially in rural areas; reason for which the present study was carried out, which focuses, which allows to identify the influential factors of marital relationships in adolescents., so as to design strategies that allow to diminish this situation in schools. A qualitative approach is assumed, 31 students from the eighth, ninth and tenth grades of the Josefa Romero de la Cruz Educational Institution were taken as a sample. Among the findings found there is a lack of guidance in the adolescence stage for decision making as opposed to starting a conjugal life, for what the teachers must strengthen in the pedagogical practice on topics inherent to education and training in education issues sexual.

Keywords: Conjugal relations, Adolescence, education sexual.

\footnotetext{
1 Este artículo ha sido derivado del Programa de Fortalecimiento de la Cultura Ciudadana y Democrática CT+I a través de la IEP apoyada en TIC en el Departamento de Magdalena: CICLON.

2 Líder de investigación del grupo "Investigadores del Rosario".

3 Docentes pertenecientes al grupo de investigación "Investigadores del Rosario", de la IED Josefa María Romero de la Cruz, ConcordiaMagdalena.
}

- The author; licensee Universidad de la Costa - CUC. 


\section{Introducción}

Vivimos en un mundo en el que coexisten variedad de sistemas sociales, que no son necesariamente homogéneos, sino más bien heterogéneos, y que tienen características marcadamente modernas, esto justifica lo que hoy se conoce como libre forma de pensar y actuar, sobre todo en las etapas de desarrollo máximo como lo es la adolescencia (Herrera, Certain y Calderón. 2014).

En la época actual, indudablemente existen grandes problemas que aquejan a la sociedad, dentro de los cuales se destacan los cambios en los estilos de vida, el crecimiento acelerado lo cual ha generado disfunciones sociales y uno de ellos es el inicio de las relaciones conyugales en la adolescencia. La juventud es una etapa de oportunidades y cambios en donde los jóvenes potencian un sinnúmero de capacidades como lo son: observar, aprender, experimentar, utilizar el pensamiento crítico, expresar su libertad creativa, y participar en procesos sociales y políticos, por lo que desarrollar de manera adecuada todas estas capacidades debe ser la prioridad en todas las sociedades actuales.

El objetivo del proyecto fué diseñar identificar los factores determinantes en las relaciones conyugales en adolescentes de La IED Josefa María Romero de La Cruz.

\section{Adolescencia}

De acuerdo a la Organización Mundial de la Salud define la adolescencia como "el periodo de crecimiento y desarrollo humano que se produce después de la niñez y antes de la edad adulta, entre los 10 y los 19 años”. Básicamente Se trata de una de las etapas de transformación más importantes en la vida del ser humano, que se caracteriza por un ritmo acelerado de crecimiento y de cambios. Esta periodo de crecimiento y desarrollo viene acompañada por diversos procesos biológicos. El comienzo de la pubertad marca el pasaje de la niñez a la adolescencia (Mañeru, 1999).
"Los determinantes biológicos de la adolescencia son prácticamente universales; en cambio, la duración y las características propias de este periodo pueden variar a lo largo del tiempo, entre unas culturas y otras, y dependiendo de los contextos socioeconómicos. Así, se han registrado durante el pasado siglo muchos cambios en relación con esta etapa vital, en particular el inicio más temprano de la pubertad, la postergación de la edad del matrimonio, la urbanización, la mundialización de la comunicación y la evolución de las actitudes y prácticas sexuales "(Organización Mundial de la Salud-OMS)

\section{Relaciones Conyugales en Adolescentes}

Es importante mencionar que la relación conyugal es aquella que enmarca la unión afectiva, espiritual y carnal entre dos personas, sin concebir el matrimonio. (De Miguel, 2016).Una relación conyugal, según Muñoz T; Jiménez I; y Moreno, M. (2008). También se da cuando dos personas que no necesariamente como se ha venido observando desde la antigüedad "deben" ser de diferentes sexos, sino que por el contrario pueden ser del mismo, es dada a conocer cuando hay atracción mutua y son autónomos de decidir qué tipo de vínculo, en donde intervienen diversos factores, (familia, edad, amigos etc) (Morales citando a Diaz-Loving y Rivera. 2010). Se puede inferir que una relación conyugal se puede dar en personas con discapacidad, tal cual Araque y Suárez (2017) expresan como en la antigüedad, la discapacidad era asumida como una "anormalidad" producto del castigo divino, en otros casos, como una consecuencia de los pecados cometidos por los familiares de la persona que presentaba esta condición.

A los jóvenes les llama la atención el tema de relaciones de pareja porque esta influye en su vivencia cotidiana y sus proyectos de vida, ya sea que hayan tenido 
o no alguna experiencia en este campo. La búsqueda de una pareja, difiere de cada persona, en donde se pueden observar acciones específicas de acorde a las situaciones. Resulta determinante inferir que las relaciones afectivas son propias de la adolescencia, por esto se le cobra gran importancia (Dávila y Goicovic, 2002; Molina, 2001).

Las consolidaciones de relaciones por parte de los jóvenes se originan por diversos motivos, algunos buscan pareja como apoyo, para compartir momentos, para divertirse, de acuerdo al contexto en donde se desenvuelva. Es la familia, los amigos y las relaciones de pareja en donde los individuos más comparten y socializan (Pérez y Valdez, 2003). Es la juventud la que es entendida tal como lo afirma Weiss (2004),

"Un tiempo importante de socialización y a la vez de subjetivación o individuación.

Para ello, la relación con amigos y novios es primordial".

Es importante mencionar que el inicio de una relación conyugal a temprana edad, aumenta la posibilidad a gran escala de tener hijos de forma temprana, y en muchos de los casos se convierte en una barrera para terminar la educación secundaria, y además tiene fuertes significativos impactos en la inclusión laboral sobre todo de las mujeres jóvenes (Monjas, Sureda y García, 2008; Olavarría, 2004 Monjas, Sureda y García, 2008).

\section{La sexualidad en los Adolescentes}

Toda manifestación de la sexualidad va más allá de las respuestas genitales, manifestándose en las diarias conductas de relación con personas de cualquier sexo, fuerzas fisiológicas, emocionales, intelectuales, sociales y culturales que condicionan la sexualidad de manera muy importante, especialmente durante la infancia, niñez $\mathrm{y}$ adolescencia, influyendo sobre posibles actitudes y conductas de la vida adulta, que están sujetas a cambios de manera constante como resultado de la experiencia y el aprendizaje. Mendoza et al (2012). Son las relaciones románticas, las que favorecen en gran medida el desarrollo sano de la sexualidad, se constituye como una tarea importante en esta etapa de la vida del adolescente.

"Teniendo en cuenta las consecuencias de la actividad sexual durante la adolescencia la literatura plantea que estas pueden ser físicas, psicológicas y sociales. Las físicas, como el embarazo no deseado y las Enfermedades de Transmisión Sexual, y las sociales, como la sanción social o el reconocimiento y la aprobación por parte de la pareja, el grupo de iguales o la familia” (Muñoz, Jiménez y Moreno, 2008).

\section{Relaciones interpersonales}

En la vida del adolescente juega un papel importante la amistad, puesto que les ofrece una red de apoyo social y emocional que le ayuda a alcanzar mayor independencia, enfrentándose a situaciones en las que deben tomar decisiones para sus vidas sin tener la mayor experiencia de un adulto y en los cuales, casi todos los desarrollos que ha adquirido y alcanzado teniendo en cuenta su participación social, y aquí se cruzan los sentimientos, pues se espera que al establecer relación con alguien sea para buenos momentos, pero no en todos los casos, es en las relaciones afectivas donde aparecen alegrías y tristezas frecuentemente (Lorente, 2007).

Es la familia ha sido considerada como la primera institución social humana, la cual sobrevivirá de una u otra forma mientras exista la especie (Tomé, 2001; Paidós, 2000; Olavarría, 2004; Icaria, 2010 y Tomé, 2001). El termino de familia según Gómez y Villa (2014) se ha defendido como el lugar primordial donde se comparten y gestionan los riesgos sociales de sus miembros. La familia es un núcleo 
compuesto por dos o más personas y se constituyen por padre e hijos a partir de la unión de una pareja en comunidad a partir de la unidad de una pareja, este grupo dentro de la sociedad requiere de una prioritaria atención. .

Los adolescentes esperan y requieren siempre de la mayor atención por parte de los padres o cuidadores, en todos los momentos, escolares, familiares, de recreación, de trabajo entre otros. Que exista un contacto directo de supervisión, apoyo, respaldo y seguimiento (Mendizábal y Anzures 1999).

\section{Metodología}

La presente investigación es soportada desde el paradigma socio - critico, que busca comprender e interpretar la realidad, a partir del estudio de los fenómenos, en su contexto. El alcance de la investigación es descriptiva (Martínez 2006).

\section{Escenario y actores}

El contexto donde se desarrolla la investigación es en la Institución Educativa Josefa Romero de la Cruz. Se decidió con estudiantes de $9^{\circ}, 10^{\circ}$ y $11^{\circ}$, con edades de 12-17 años ya que son ellos los que según diagnóstico realizado en la comunidad quienes inician vida conyugal desde edades tempranas. Se contó con la participación de (31) estudiantes.

\section{Técnicas e instrumentos de recolección de la información}

Dado el tipo de investigación cualitativa y las características del escenario de investigación se decide utilizar como técnicas de recolección: La entre- vista. Estructurada y la observación directa, registrada en un diario de campo.

\section{Procedimiento}

Se construyeron los contenidos de acuerdo de acuerdo a la problemática estudiada. Luego fue enviada a pares expertos para su respectiva validación. Una vez validados se diseñó un instrumento para ser sometido a una prueba piloto, tomando los aportes más significativos de los aspectos evaluados.

Culminada la prueba muestral se envió de nuevo a los jueces expertos, quienes hicieron las correcciones y recomendaciones pertinentes. Finalmente se procede a realizar la aplicación a la población objeto de estudio. El diseño de la Entrevista estructurada se formuló de tal manera que se obtuviera información precisa sobre el objeto de investigación, para la identificación de factores que inciden en el inicio de relaciones conyugales en adolescentes.

El diseño de las preguntas, tuvo una guía que permitiera elaboración definitiva del cuestionario y del plan operativo de la entrevista basándose en propósitos específicos de la investigación, se realizó una validación presentada a expertos y fue aplicada a estudiantes de octavo, noveno y décimo de Básica secundaria de la IED Josefa María Romero de la Cruz.

\section{Resultados}

A continuación en la tabla 1 se presentan lo hallazgos encontrados en el estudio. 


\begin{tabular}{|c|c|c|c|}
\hline $\begin{array}{c}\text { Categoría de } \\
\text { estudio }\end{array}$ & $\begin{array}{c}\text { Pregunta } \\
\text { orientadora }\end{array}$ & Discurso de los actores & Articulación y sistematización teórica \\
\hline
\end{tabular}

Relaciones Conyugales
¿Para usted que son las relaciones conyugales?
"Para mi las relaciones conyugales son cuando unas parejas deciden vivir sin casarse por la iglesia o por lo civil. Ejemplo: si una chica y un chico se salen a vivir eso es una relación conyugal", "Las relaciones conyugales son aquellas cuando una persona se va a vivir con otra sin haber cruzado el matrimonio", "para mi las relaciones conyugales son las personas que se casan a temprana edad sin importar las consecuencias de las relaciones conyugales", "para mí las relaciones conyugales son relaciones que no dependen de un anillo ni un escrito, mejor dicho, deciden estar en unión libre", "son aquellas personas que se salen sin pensar las relaciones que van a tener en el futuro", "son aquellas donde no pueden, o pueden confirmar un matrimonio o una relación. Donde las personas viven y no tienen que mostrar o confirmar matrimonio", "son los jóvenes que se casan o se salen sin estar comprometidos o sin algún permiso de los padres o de la iglesia”, "para mí las relaciones conyugales son cuando los jóvenes se casan sin estar comprometidos, sino que pueden ser novios, y de repente se la sacan sin tener permiso de los padres o de la iglesia", "para mí las relaciones conyugales son los jóvenes por decirlo así se salen a veces a escondidas para formar una relación conyugal, casados sin estar comprometidos", "Las relaciones conyugales para mi es cuando las personas se casan sin el permiso de sus padres, cuando se casan a escondidas".
De acuerdo a los hallazgos encontrados es posible inferir que los estudiantes en su mayoría definen las relaciones conyugales como ese vínculo que establecen entre dos personas para compartir una vida amorosa y sentimental, pero, sin estar legalmente casados. Desde esta mirada los resultados dejan ver claridad en la definición, y marcando la diferencia de una unión marital debidamente concebida, tal como lo expone De Miguel, (2016) .La relación conyugal es aquella que enmarca la unión afectiva, espiritual y carnal entre dos personas, sin concebir el matrimonio. Así mismo, los resultados dados por los estudiantes incluyen las relaciones sexuales como componente importante al iniciar una vida conyugal, tal como lo manifiestan a continuación:

"Las relaciones conyugales son aquellas que se dan entre personas a temprana edad y empiezan una vida sexual con ella, sin tener idea de lo que es afrontar una relación conyugal”, "una relación conyugal es irse a vivir con una persona, y empezar una vida sexual con ella". Las relaciones conyugales se les puede concebir según Morales, D. (2016) como "una asociación característica entre dos o más personas que involucra una interacción y que puede tener y que lleva como fin la consolidación de una relación amorosa, en donde la sexualidad cobra gran importancia en su plenitud y poderla experimentar abiertamente, es una de las principales razones para que los adolescentes inicien una vida marital". Otros hallazgos significativos son los que se encontraron a raíz de la siguiente apreciación: "Es cuando una persona se convierte en pareja sentimental de otra, ya sea del mismo sexo o del sexo contrario". responsabilidad de ambos" (Morales, D. 2016. Citando a Diaz-Loving y Rivera. 2010). 


\begin{tabular}{|c|c|c|c|}
\hline $\begin{array}{c}\text { Categoría de } \\
\text { estudio }\end{array}$ & $\begin{array}{c}\text { Pregunta } \\
\text { orientadora }\end{array}$ & Discurso de los actores & Articulación y sistematización teórica \\
\hline $\begin{array}{l}\text { Relaciones } \\
\text { Conyugales }\end{array}$ & $\begin{array}{l}\text { ¿Para usted } \\
\text { que son las } \\
\text { relaciones } \\
\text { conyugales? }\end{array}$ & & $\begin{array}{l}\text { Lo que reafirma que una relación conyugal, } \\
\text { también se da cuando dos personas que } \\
\text { no necesariamente como se ha venido } \\
\text { observando desde la antigüedad "deben" ser } \\
\text { de diferentes sexos, sino que por el contrario } \\
\text { pueden ser del mismo, "se conocen hasta } \\
\text { llegar al punto de atraerse mutuamente, } \\
\text { mientras pasa el tiempo ellos deciden si } \\
\text { inician un vínculo y según se da la misma, } \\
\text { ellos pueden formalizar o terminar con } \\
\text { dicha interacción, esto va a depender de } \\
\text { muchos factores y elementos que se den } \\
\text { en la forma como se tratan debido a que } \\
\text { el mantenimiento de la misma es. Son los } \\
\text { estudiantes los que finalmente describen } \\
\text { la relación conyugal como la unión de dos } \\
\text { personas que no contemplan un matrimonio } \\
\text { formalmente establecido, sino que por el } \\
\text { contrario deciden vivir una experiencia } \\
\text { marital sin concepción de terceras personas, } \\
\text { y que inicialmente lo hacen de manera } \\
\text { oculta y pre-acordada. }\end{array}$ \\
\hline
\end{tabular}

\begin{tabular}{|c|c|c|c|}
\hline $\begin{array}{l}\text { Relaciones } \\
\text { conyugales }\end{array}$ & $\begin{array}{l}\text { ¿La } \\
\text { formación } \\
\text { académica de } \\
\text { los padres es } \\
\text { pertinente } \\
\text { a la hora da } \\
\text { orientar a los } \\
\text { estudiantes } \\
\text { frente al } \\
\text { inicio de } \\
\text { una relación } \\
\text { conyugal a } \\
\text { temprana } \\
\text { edad? }\end{array}$ & $\begin{array}{l}\text { "Si, porque los padres deben } \\
\text { orientar y dar consejos ara que } \\
\text { prevengan una relación conyugal } \\
\text { a temprana edad", "si, porque } \\
\text { algunos padres no ayudan } \\
\text { a reflexionar sobre casarse a } \\
\text { temprana edad y por mucho que } \\
\text { no estudien siempre nos dan } \\
\text { consejos para no cometer errores", } \\
\text { "para mi mis padres me dan una } \\
\text { buena orientación porque ellos } \\
\text { nos dicen que todavia no tenemos } \\
\text { la edad adecuada para tener } \\
\text { una relación, que disfrutemos } \\
\text { la vida y no nos arrepintamos } \\
\text { más adelante", "para mi me dan } \\
\text { una buena orientación porque } \\
\text { ellos nos dicen que disfrutemos } \\
\text { la vida porque todavia estamos } \\
\text { muy pequeñas para tener } \\
\text { relaciones", "si, porque los padres } \\
\text { deben orientar a los estudiantes } \\
\text { o a sus hijos para no coger ese } \\
\text { mal camino y nunca llevarle la } \\
\text { contraria a su padres, porque } \\
\text { ellos lo que quieren es un bien } \\
\text { para nosotros". }\end{array}$ & $\begin{array}{l}\text { Los anteriores resultados permiten mostrar } \\
\text { la importancia de la educación de los } \\
\text { padres al momento de orientar a sus hijos } \\
\text { adolescentes. Los estudiantes infieren que } \\
\text { resulta relevante su formación, pues ellos } \\
\text { orientan ante las decisiones que se puedan } \\
\text { tomar, les comparten sus historias de vidas } \\
\text { y además siempre buscan el bienestar de } \\
\text { vida y la reducción de inadecuadas acciones, } \\
\text { en otras palabras son los padres los que una } \\
\text { pieza clave en el proceso de crecimiento de } \\
\text { los estudiantes. } \\
\text { Tal como lo sustentan Simpson (2004). Son } \\
\text { los padres quienes juegan un papel crucial, } \\
\text { irreemplazable en la educación de los hijos. } \\
\text { Se estima que cuando lo adolescentes } \\
\text { están en el tránsito de una etapa a otra, } \\
\text { la toma de decisiones, las orientaciones, } \\
\text { acompañamiento y demás, es necesario y } \\
\text { pertinente para reducir comportamientos } \\
\text { impulsivos, tras el desconocimiento de } \\
\text { ciertas situaciones. } \\
\text { En el inicio o no de una vida conyugal en la } \\
\text { adolescencia, autores como Verano (2002) } \\
\text { afirman que la formación académica de los } \\
\text { padres es un factor determinante, pues se } \\
\text { estima que "padres que culminaron sus } \\
\text { estudios, cuentas con diversas estrategias } \\
\text { para acompañar a sus hijos en la toma de } \\
\text { decisiones, en brindarles conocimientos } \\
\text { sobre las consecuencias positivas y negativas } \\
\text { de una relación de pareja". }\end{array}$ \\
\hline
\end{tabular}




\begin{tabular}{|c|c|c|c|}
\hline $\begin{array}{c}\text { Categoría de } \\
\text { estudio }\end{array}$ & $\begin{array}{c}\text { Pregunta } \\
\text { orientadora }\end{array}$ & Discurso de los actores & Articulación y sistematización teórica \\
\hline $\begin{array}{l}\text { Relaciones } \\
\text { conyugales }\end{array}$ & $\begin{array}{l}\text { ¿La } \\
\text { formación } \\
\text { académica de } \\
\text { los padres es } \\
\text { pertinente } \\
\text { a la hora da } \\
\text { orientar a los } \\
\text { estudiantes } \\
\text { frente al } \\
\text { inicio de } \\
\text { una relación } \\
\text { conyugal a } \\
\text { temprana } \\
\text { edad? }\end{array}$ & 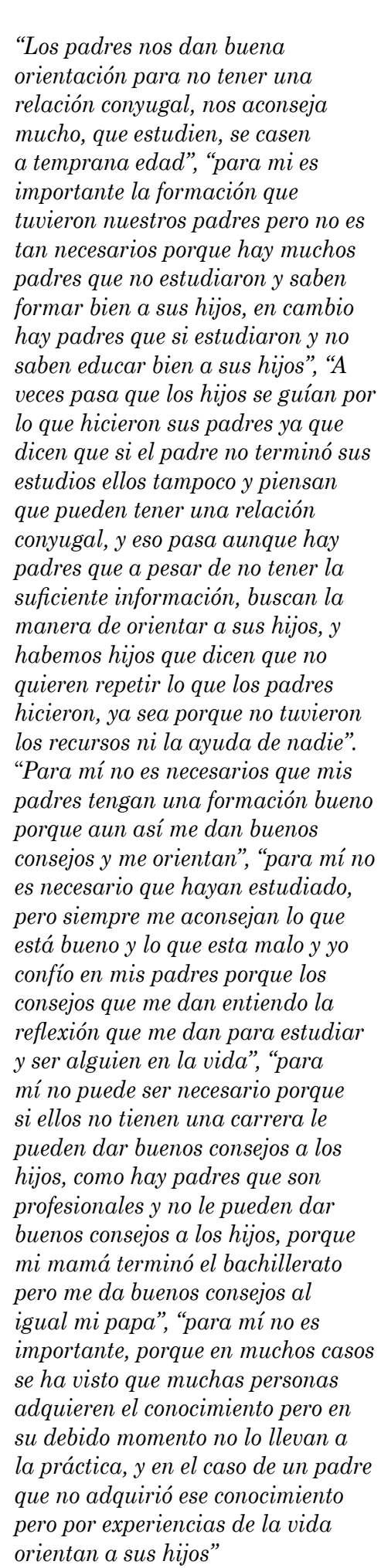 & 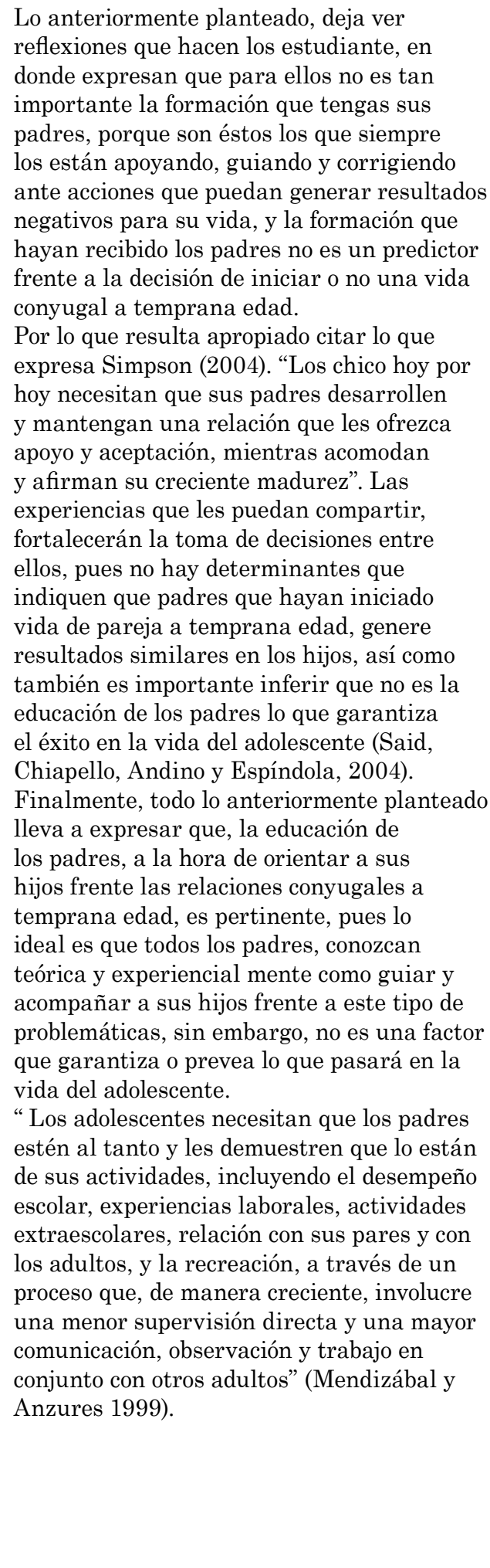 \\
\hline
\end{tabular}




\begin{tabular}{|c|c|c|c|}
\hline $\begin{array}{c}\text { Categoría de } \\
\text { estudio }\end{array}$ & $\begin{array}{c}\text { Pregunta } \\
\text { orientadora }\end{array}$ & Discurso de los actores & Articulación y sistematización teórica \\
\hline $\begin{array}{l}\text { Relaciones } \\
\text { conyugales }\end{array}$ & $\begin{array}{l}\text { ¿La } \\
\text { formación } \\
\text { académica de } \\
\text { los padres es } \\
\text { pertinente } \\
\text { a la hora da } \\
\text { orientar a los } \\
\text { estudiantes } \\
\text { frente al } \\
\text { inicio de } \\
\text { una relación } \\
\text { conyugal a } \\
\text { temprana } \\
\text { edad? }\end{array}$ & $\begin{array}{l}\text { "No, pero eso no significa que ellos } \\
\text { no puedan orientar y ayudar para } \\
\text { que nosotros no cometamos tantos } \\
\text { errores y tener una orientación } \\
\text { clara", "la verdad pienso que } \\
\text { la formación académica no es } \\
\text { tan importante, puesto que en } \\
\text { muchos casos los padres que } \\
\text { son profesionales y expertos } \\
\text { en la vida conyugal, tienden a } \\
\text { tener un desastre en el hogar, y } \\
\text { en cambio los otros que no son } \\
\text { estudiados, tienden a educar } \\
\text { mejor a sus hijos", "para mí no } \\
\text { es necesariamente que los padres } \\
\text { sean bachilleres o profesionales, } \\
\text { porque mis padres me dan buenos } \\
\text { consejos como que estudie, que los } \\
\text { estudios es lo único que nos va a } \\
\text { servir para la vida y no dejar de } \\
\text { estudiar". }\end{array}$ & \\
\hline
\end{tabular}

\begin{tabular}{|c|c|c|c|}
\hline Adolescencia & $\begin{array}{l}\text { ¿Cuál cree } \\
\text { usted que es } \\
\text { el papel que } \\
\text { debe asumir } \\
\text { la escuela } \\
\text { frente al } \\
\text { inicio de } \\
\text { relaciones } \\
\text { conyugales } \\
\text { en } \\
\text { adolescentes? }\end{array}$ & $\begin{array}{l}\text { "Orientar y dar charlas a los } \\
\text { estudiantes, y sobre todo aconsejar } \\
\text { y aclarar los riesgos que se } \\
\text { corren al inicio de las relaciones } \\
\text { conyugales", "para mi la escuela } \\
\text { tendría que dar muchas charlas } \\
\text { sobre las relaciones conyugales", } \\
\text { "para mi la escuela nos tendría } \\
\text { que dar charlas, comité de } \\
\text { relaciones conyugales en la } \\
\text { adolescencia y muchas cosas", } \\
\text { "pues para mi dar charlas o } \\
\text { presentar actividades mostrando } \\
\text { algunos casos para que nosotros } \\
\text { nos orientamos mejor al escoger } \\
\text { una buena o mala decisión", "yo } \\
\text { diría que la institución debería } \\
\text { orientarnos un poco sobre las } \\
\text { relaciones conyugales”, }\end{array}$ & $\begin{array}{l}\text { Las respuesta manifestadas por los } \\
\text { estudiantes hacen notoria la necesidad de } \\
\text { que el colegio los forme en capacitaciones } \\
\text { y talleres sobre las relaciones conyugales, } \\
\text { los cuidados y el conocimiento que se debe } \\
\text { tener al iniciar una vida en pareja, así } \\
\text { como mostrarle situaciones que permitan } \\
\text { la confrontación, promuevan la crítica y la } \\
\text { autonomía para tomar decisiones frente a la } \\
\text { temática. } \\
\text { Tal como lo expone Monserrat (s.f). La } \\
\text { escuela la que constituye uno de los lugares } \\
\text { en el que los adolescentes ocupan la mayoría } \\
\text { de su tiempo, se convierte en un entorno } \\
\text { habitual de interacción de los estudiante, es } \\
\text { donde ellos van des cubriendo capacidades, } \\
\text { habilidades, asumen retos y desafíos propios } \\
\text { de la edad. } \\
\text { La vida adolescente conlleva a la toma } \\
\text { de múltiples decisiones, y lo esperado es } \\
\text { que cada individuo disfrute de sus etapas } \\
\text { y el colegio les potencie capacidades que } \\
\text { promuevan la autonomía, y libertad ante la } \\
\text { toma de decisiones. Por lo anterior algunos } \\
\text { estudiantes manifiestan lo siguiente: }\end{array}$ \\
\hline
\end{tabular}




\begin{tabular}{ccc}
\hline $\begin{array}{c}\text { Categoría de } \\
\text { estudio }\end{array}$ & $\begin{array}{c}\text { Pregunta } \\
\text { orientadora }\end{array}$ & Discurso de los actores \\
\hline
\end{tabular}

Articulación y sistematización teórica

Por lo tanto es en el colegio, las prácticas educativas constituyen parte fundamental de la mediación social necesaria para orientar y apoyar el paso de los adolescentes a la vida

"para mí la escuela que tenga buena orientación para que los jóvenes salgan adelante", "para mi el papel de la escuela es de seguir orientando a los jóvenes, darles una guía de seguir estudiando", "para mí la escuela debe seguir

¿Cuál cree usted que es el papel que debe asumir la escuela

Adolescencia frente al inicio de relaciones conyugales en adolescentes? orientando a los estudiantes para que no se den casos de estos en la escuela", "deben orientarnos por medio de sus conocimientos los docentes, ya que hablamos de esto en casa y lo fortalecemos en la escuela", Orientar, y sobre todo los profesores darle charla a los estudiantes y junto a sus padres orientar", "no permitir las relaciones conyugales porque de pronto no tienen una autorización de los padres, o donde los padres no estén de acuerdo". adulta, y su inclusión como miembros activos de la sociedad, con derechos y deberes de igualdad y participación, no importando las condiciones, siempre y cuando estas no afecten la vida escolar de los estudiantes. (Castillo 2013 Vargas, Ibáñez \& Feliz 2005). Otros hallazgos dejan ver que, en el acto educativo, en la consecución de procesos de enseñanza aprendizaje, y en problemáticas propias de la etapa adolescente, no solo median y actúan los estudiantes y docentes, pues son los padres los que ejercen de igual manera un papel importante.

Las características del contexto familiar influyen de manera directa en los comportamientos que asumen los adolescentes, las relaciones padres e hijos resultan determinantes, la participación constantes a través de redes académicas, y escuelas de padres, deben expandirse como herramientas de apoyo, pues contribuye y facilitan a la formación del auto concepto, identidad personal, autoestima, las expectativas, motivaciones, experiencias, y niveles de superación de los jóvenes Monserrat (s.f).

Pienso que es bueno, porque aunque poco trato temas de esta magnitud siempre están ahí para lo que yo necesite, y como en toda relación hay problemas esta no es la excepción, pero siempre trato de ser lo mejor posible para estar bien", "pues es una buena relación, pero también existen ocasiones en la que por distintas razones pueden existir disgustos pero siempre hacemos lo posible para solucionarlos", "mi relación con mis padres es buena, ya que hay comunicación, algunas veces se ve la confianza, pues mis padres son muy comprensivos conmigo y además de eso nos brindan un amor y apoyo incondicional”,
Los hallazgos encontrados permiten afirmar según lo expresado por los estudiantes que la relación con sus padres es buena, porque los orienta, apoyan, se comunican abiertamente, y les brindan buenos consejos, para que no tomen decisiones equivocas. Esto se fortalece tomando como referencia lo que expone Mendizábal \& Anzures (1999). Son las redes de apoyo las que promueven acciones de solidaridad que faciliten el cuidado y crianza de los hijos y compensen las deficiencias del sistema familiar. Están constituidas por miembros de la familia extensa, amigos y vecinos que puedan proporcionar ayuda. La comprensión mutua y la comunicación constituyen elemento indispensable para identificar y resolver los problemas, desafortunadamente durante la adolescencia esta última se ve sumamente afectada (Mendizábal \& Anzures.1999). 


\begin{tabular}{cl}
\hline $\begin{array}{c}\text { Categoría de } \\
\text { estudio }\end{array}$ & $\begin{array}{c}\text { Pregunta } \\
\text { orientadora }\end{array}$ \\
\hline Adolescencia & $\begin{array}{l}\text { Actualmente } \\
\text { como es la }\end{array}$ \\
& relación con \\
& sus padres \\
& ¿Buena \\
& o mala? \\
& ¿Porque?
\end{tabular}

\author{
Discurso de los actores
}

"mi relación con mis padres es muy buena, siempre ha sido así, tenemos una buena comunicación, ante todo confianza, ellos me orientan y me explican sobre los peligros a los que estoy expuesta y las consecuencias a las que me pueden llevar mis actos", "buena, mis padres siempre me sacan un tiempo para hablarme sobre todas las cosas que son malas y buenas para mi vida”, "actualmente mi relación con mis padres es buena, porque hay momentos en que necesito de su orientación y de su confianza, más que todo la distancia te hace amarlos más, y tener la necesidad de contarle mis cosas", "pues es buena porque yo no vivo con mi papá, pero si tengo un padrastro y se lleva bien, hay comunicación entre ellos no pelean, hay comunicación y nos entendemos", "mi relación con mis padres es muy buena porque me dicen que es lo que no debo hacer y me dan la confianza necesaria”. "Pues mi relación con mis padres, es mala porque ellos no viven conmigo se separaron y no están a mi lado para darme un buen consejo para mi vida no los tengo y ellos no se dan a querer", la relación con mis padres no es tan bien porque casi nunca me hablan de relaciones sexuales", ni buena, ni mala. Regular. Porque muchas veces nos dan consejos, pero cuando realmente lo necesitamos no", regular, porque hay veces que mis padres no me saben tratar bien, y lo malo de todo es que no me orientan sobre las relaciones sexuales a temprana edad, ni de embarazos no deseados, ni cómo puedo controlar cuando estoy con un grupo de amigos que sean mala influencia”.
Articulación y sistematización teórica

Lo anterior deja ver que los estudiantes manifiestan tener una mala relación con sus padres, pese a que afirman que ni buena ni mala. Solo describen factores negativos haciendo alusión a la desestructuración del núcleo familiar, a la ausencia de diálogos sobre la sexualidad, (propio de la edad) y a la falta de orientación y apoyo. De acuerdo a lo anterior Mendizábal y Anzures (1999). Exponen: Los inconvenientes en la relación padres-hijos, parten principalmente alrededor de tres áreas: la autoridad, la sexualidad y los valores. La comunicación entre los jóvenes y sus padres frecuentemente se caracteriza por una mínima comunicación y una expresión afectiva negativa, que suelen ser un recurso negativo a la hora de llevar una buena conducta.

La adolescencia es una etapa de exploración de la sexualidad recientemente descubierta, lo que conlleva a una serie de conflictos acerca de los valores sexuales y su expresión. No cabe duda que existe una dificultad de los padres a la hora de socializar temas de orden sexual. Son los padres quienes asumen posturas incomodas y temen a las reacciones negativas de los hijos frente a temas como estos, desconociendo como asumen los adolescentes esta etapa. Por lo tanto, uno de los principales canales que los padres deben afianzar y fortalecer con sus hijos es la comunicación, en donde se potencia el afecta, fortalecen los valores como la confianza y se acompaña en la toma de decisiones complejas como la de iniciar la vida sexual (Mendizábal y Anzures.1999).

Fuente: elaboración propia. 


\section{Discusión}

Los adolescentes reconocen lo que significa una relación conyugal en la adolescencia y logran enmarcarla como una problemática o conducta negativa para ellos, pues manifiestan que esta se caracteriza por la unión marital de una pareja que no consolida un matrimonio y que lo hace a escondidas. A sí mismo, toma en cuenta el componente sexual como uno de los principales motivos por el cual los adolescentes toman esta decisión, explicando que es una opción para poder ejercer la vida sexual de una manera libre y sin limitaciones.

Son las relaciones conyugales a temprana edad una de las más grandes problemáticas que se presentan en la IED Josefa María Romero de la Cruz, y aunque el estudiante logre definirla, se aleja de las consecuencias que esto pueda acarrear, pues comentan que por lo general las decisiones frente a iniciar una vida de pareja no son debidamente razonadas y consultadas, sino que por el contrario, son impulsos del momento que posteriormente llevan en la mayoría de los casos al arrepentimiento.

La mayoría de los estudiantes reconocen que la educación de los padres juega un papel importante a la hora de orientar a los adolescentes frente a una vida conyugal a temprana edad, sin embargo, explican que aunque algunos padres no estén formados académicamente, siempre están apoyando, guiando, retroalimentándolos en situaciones que puedan generar problemas en la vida adolescente.

Por otro lado, otro grupo de estudiantes, afirma que no es tan importante la formación de los padres, porque según ellos, en la mayoría de los casos, son los padres los que desean una vida satisfactoria para sus hijos, que aprovechen las etapas propias de la edad, enfrentándo- las y disfrutándolas, y no se requiere de una formación académica para brindar este tipo de acompañamiento.

La mayoría de los adolescentes existe una buna comunicación con los padres, afirmando que son ellos los que siempre están en disposición de escucha, de brindar consejos, de hablarle sobre temas que son novedosos para ellos y que sobre todo les brindan cuidados frente a situaciones de riesgo. Por otro lado una minoría de los estudiantes tiene una relación negativa con sus padres, estableciendo como primer factor el origen de un hogar desestructurado (madres y/o padres ausentes), poca tolerancia a comportamientos de rebeldía manifestados por los jóvenes, ausencia en el establecimiento de normas y autoridad en el hogar y baja comunicación asertiva entre los miembros.

Determinar la responsabilidad de la escuela frente a programas que tienden a fortalecer la situación presentada por os adolescentes de la IED Josefa María Romero de la Cruz. El estudiante manifiesta que es la escuela un lugar de formación, no solo académica, sino además de crecimiento personal integral, por lo que reconoce la importancia y el papel de esta frente al inicio de relaciones conyugales a temprana edad. Identifican que son los maestros, personas que pueden apoyar, orientar, guiar, y brindar conocimientos de lo que realmente significa tener una relación conyugal a edades tempranas, las consecuencias que se pueden tener, y además brindarle información de ciertos componentes que hacen parte de una vida en pareja y como deben manejarse.

Finalmente hacen el llamado a la escuela a que les facilite talleres y capacitaciones sobre temáticas como: Sexualidad, embarazos no deseados, enfermedades de transmisión sexual, drogadicción, influencia de pares, entre otras. 


\section{Referencias}

Álvarez, A. (2014). Las relaciones sexuales desde la mirada de los adolescentes. digitalciencia@uaqro. 31(6). 1-8. Recuperado de https://www.researchgate.net/publication/303064877_Las_relaciones_sexuales_desde_la_mirada_de_los_ adolescentes

Araque, F. y Suárez, O. (2017). Equidad Ética-Jurídica de la Ciencia, para la Emancipación del Conocimiento y los Saberes. Jurídicas CUC, 13, (1). 97-120. DOI: http://dx. doi. org/10.17981/jurid cuc.13.1.2017.5

Avendaño, I., Cortés, O. y Guerrero, H. (2015). Competencias sociales y tecnologías de la información y la comunicación como factores asociados al desempeño en estudiantes de básica primaria con experiencia de desplazamiento forzado Diversitas: Perspectivas en Psicología, 11(1). 13-36. Recuperado de https://www.redalyc. org/pdf/679/67943296001.pdf

Cifuentes, G. (2013). Desarrollo Constitucional, Legal y Jurisprudencia del Principio de Rigor Subsidiario. Jurídicas CUC, 9(1), 345 - 384.

Cortés-Peña, O. (2016). Sustainable development in synergistic relationship with pro-ambient behavior and fair trade. International Journal of Management Science And Operation Research (Ijmsor), 1(1), 54-58. Retrieved from http://ijmsoridi.com/ index.php/ijmsor/article/view/78

Eresta, M. (2013). Relaciones afectivas y Sexualidad en la Adolescencia. Madrid: Liga Española de Educación. Recuperado de http://www.ligaeducacion.org/documentos/ investigaciones/relaciones-afectivasy-sexualidad-en-la-adolescencia.pdf
Fernandez, I. (2002). Modelos de relación conyugal. 14 casos de mujeres de diferentes. [Trabajo de maestría]. Valle del Cauca, Calí. Recuperado de http://cognitiva.univalle.edu.co/archivos/grupo\%20cultura/recursos/ Informe_Adriana.pdf

Guerrero, H.(2015) El portafolio: una herramienta facilitadora del cambio en la educación superior desde la práctica didáctica. Zona Próxima, 22. http://dx.doi.org/10.14482/ zp. 22.6340

Herrera, B., Certain, R. y Calderón, M (2014).La norma hipotética fundamental desde el Paradigma Interpretativo. Justicia Juris, 10(2), 17-27. http://dx.doi.org/10.15665/ rj.v10i2.324

Icaria, E. (2010). La igualdad también se aprende: cuestión de coeducación. Madrid: Narcea.

Lorente, M. (2007). Violencia de género, educación y socialización: acciones y reacciones. Revista de Educación, 342(1), 19-35. Recuperado de http:// www.revistaeducacion.mec.es/ re342/re342_02.pdf

Mañeru, A. (1999). Autoridad femenina en educación. Kikiriki, 54, 26-31.

Mañeru, A. (2007). La práctica de la autoridad en las relaciones. Crítica, 57(943). 55-59. Recuperado de https://dialnet.unirioja.es/servlet/ articulo?codigo $=2516552$

Mendoza, L., Arias, M., Pedroza, M., Micolta, P., Ramirez, A., Caseres, C., Lopez, D., Nuñez, A. y Acuña, M. (2012). Actividad sexual en adolescencia temprana: problema de salud pública en una ciudad colombiana. Rev Chil Obstet Ginecol. 77 (4). 271279. http://dx.doi.org/10.4067/S071775262012000400006 
Meza, A., Arrieta, M. y Noli S. (2018). Análisis de la conciliación extrajudicial civil en la Costa Atlántica colombiana. Jurídicas CUC, 14(1). 187-210. DOI: http://dx.doi.org/10.17981/juridcuc.14.1.2018.9

Monjas, M., Sureda, I. y García J. (2008). ¿Por qué los niños y las niñas se aceptan y se rechazan? Cultura y Educación, 20(4), 479-492.

Monserrat. (s.f). Papel de la Escuela en el desarrollo del Adolescente. Recuperado de: https://es.slideshare.net/ hellomonrodriguez/papel-de-la-escuela-en-el-desarrollo-del-adolescente

Montañés, M., Bartolomé, R., Montañés, J. y Parra, M. (2008). Influencia del contexto familiar en las conductas adolescentes. Ensayos. 1(17). 391-407 Recuperado de http://files. psicologia-2-de-secundaria.webnode. com/200000031-20fdd21fab/Dialnet-InfluenciaDelContextoFamiliarEnLasConductasAdolesc-3003557. pdf

Morales, D. (2016). Afecto y relación de pareja. [Tesis de grado]. Universidad Rafael Landívar, Quetzaltenango. Guatemala. Recuperado de http:// recursosbiblio.url.edu.gt/tesiseortiz/2016/05/42/Morales-Dulce.pdf

Muñoz, T., Jiménez, I. y Moreno, M. (2008). Reputación conductual y género en la adolescencia. Anales de psicología, 24(2), 334-340. Recuperado de http://redalyc.uaemex.mx/ pdf/167/16711589017.pdf

Navarro, E. (2004). Género y relaciones personales íntimas. En, E. Barberá e I. Martínez (coords.). Psicología y Género (171-192). Madrid: Pearson Educación.
Olavarría, J. (2004). Modelos de masculinidad y desigualdades de género. En, C. Lomas (Ed.). Los chicos también lloran. Identidades masculinas, igualdad entre los sexos y coeducación. (45- 63). Barcelon: Paidos.

Oliva, E. y Villa, V. Hacia un concepto interdisciplinario de la familia en la globalización. Justicia Juris. 10(1). 11-20. http://dx.doi.org/10.15665/rj. v10i1.295

Paidós, V. (2000). De la facultad de ver al derecho de mirar. En, M. Segarra y A. Carabí (Eds.). Nuevas masculinidades. (29-40). Barcelona: Icaría.

Said, R., Chiapello, J., Andino, G. y Espíndola, M. (2004). ¿Está la ocupación de los padres relacionada con el desempeño de sus hijos? Recuperado de http://www.unne.edu.ar/unnevie$\mathrm{j}$ a / W com2004/3-Medicina/M-010.pdf

Salazar, M. (1991). Adolescentes y sexualidad en América Latina y Colombia. Universidad Pedagogica Nacional. (22-23). http://dx.doi. org/10.17227/01203916.5199\}

Sepúlveda, A., Martínez, R., Medina, S. y Salazar, F. (2016). Propuesta de diseño de una red supply chain para la agrocadena de cacao, municipio de Viotá, Cundinamarca. International Journal of Management Sciences and Operations Research, 1(1), 3542. Recuperado de http://ijmsoridi. com/index.php/ijmsor/article/view/75

Simpson, A. (2004). Ser padres de adolescentes: Una sintesis de la investigación y una base para la acción. Washington, D.C.: Organización panamericana de la salud. Recuperado de http://hrweb.mit.edu/worklife/ raising-teens/pdfs/serpadresadol. pdf. 
Subirats, M. y Brullet, C. (1988). La transmisión de los géneros en la escuela mixta. Madrid: Ministerio de Cultura, Instituto de la Mujer.

Subirats, M. y Tomé, A. (2007). Balones fuera. Reconstruir los espacios desde la coeducación. Barcelona: Octaedro.

Tomé, A. (2001). La construcción de las identidades masculinas y femeninas en la escuela. En, N. Blanco (Coord.). Educar en femenino y en masculino. (87-98). Madrid: Akal.

Born, D., Minujín, A. y Lombardía, M. (2015). Una aproximación a la situación de adolescentes y jóvenes en américa latina y el caribe a partir de evidencia cuantitativa reciente. Ciudad de Panamá: Unicef. Recuperado de https://www.unicef.org/lac/informes/una-aproximación-la-situaciónde-adolescentes-y-jóvenes-en-américa-latina-y-el-caribe
Valdivia, C. (2008). La familia: Conceptos, cambios y nuevos modelos. La revue. Du REDIF. 1. 15-22. Recuperado de http://moodle2.unid.edu.mx/ dts_cursos_mdl/lic/DE/PF/AM/05/ cambios.pdf

Vargas, J., Ibáñez, E.y Félix, J. (2005). Evaluación de vínculo en adolescentes problemáticos. Revista Electrónica de Psicología Iztacala, 8, (3) 28-57. Recuperado de http://www.iztacala. unam.mx/carreras/psicologia/psiclin/principal.html

Vargas, E. y Barrera, F. (2002). Adolescencia, relaciones románticas y actividad sexual: Una revisión. Redalyc. org. 11. 115-134. Recuperado de https://revistas.unal.edu.co/index. $\mathrm{php/psicologia/article/}$ view/1203/1753 